UPRF-96-482

\title{
Quantum Charged Spinning Particles in a Strong Magnetic Field (a Quantal Guiding Center Theory)
}

\author{
P. Maraner \\ Dipartimento di Fisica, Università di Parma, \\ and INFN, Gruppo collegato di Parma, \\ Viale delle Scienze, 43100 Parma, Italy
}

\begin{abstract}
A quantal guiding center theory allowing to systematically study the separation of the different time scale behaviours of a quantum charged spinning particle moving in an external inhomogeneous magnetic filed is presented. A suitable set of operators adapting to the canonical structure of the problem and generalizing the kinematical momenta and guiding center operators of a particle coupled to a homogenous magnetic filed is constructed. The Pauli Hamiltonian rewrites in this way as a power series in the magnetic length $l_{B}=\sqrt{\hbar c / e B}$ making the problem amenable to a perturbative analysis. The first two terms of the series are explicitly constructed. The effective adiabatic dynamics turns to be in coupling with a gauge filed and a scalar potential. The mechanism producing such magnetic-induced geometric-magnetism is investigated in some detail.
\end{abstract}

short title: A Quantal Guiding Center Theory

PACS: 03.65.-w, 02.40+m, 52.20.Dq, 02.90.+p 



\section{Introduction}

The motion of a charged particle in a strong inhomogeneous magnetic filed is a nontrivial problem displaying a variety of very interesting phenomena ranging from chaos to phase anholonomy. Being of utmost importance in plasma physics, expecially in the study of magnetic confinement, the subject has been worked out in great detail in classical mechanics with special attention to phenomenological implications as well as to formal aspects. The canonical structure of the problem, in particular, has been deeply investigated only in a relatively recent time by R.G. Littlejohn [1] revealing the appearance of geometry induced gauge structures in the adiabatic motion of classical charged particles. Very few, on the other hand, is known about the behaviour of quantum particles in strong inhomogeneous magnetic fields, the reason being essentially that the techniques developed for classical mechanics do not easily generalize to the quantum context. Some work has been done for neutral spinning particles by M.V. Berry [2], Y. Aharonov \& A. Stern [3] and R.G. Littlejohn \& S. Weigert [4] in connections with geometrical phases, whereas a quantum treatment for charged spinning particles is still missing. It is the purpose of this paper to present what may be probably called a quantal guiding center theory in which the coupling between the spin and spatial degrees of freedom of a quantum charged spinning particle moving in a strong inhomogeneous magnetic field is systematically taken into account. This allows to extend to the quantum domain the previous classical results. Our treatment, essentially algebraic in nature, is a re-elaboration and - we believe - a simplification of the technique originally proposed by R.G. Littlejohn in classical mechanics. It is based on a different choice of non-canonical variables adapting to classical as well as quantum mechanics. Depending essentially on the canonical structure the method applies indistinctly to the classical and the quantum theory. We nevertheless focus on the quantum problem.

In order to better understand what is going on in the strong-filed regime of a quantum particle moving in an external magnetic filed it is better to first have in mind the main features of corresponding the classical problem [5]. Let us therefore to briefly consider a classical particle of mass $m$ and charge $e$ moving in a homogeneous magnetic filed of intensity $B$. As is well known the trajectory of the particle is represented by a spiral wrapping around a field line, as sketched in Fig.11a: the particle performs a uniform circular motion of frequency $\omega_{B}=e B / m c$ and radius $r_{B}=m c\left|v_{\perp}\right| / e B\left(\left|v_{\perp}\right|\right.$ is the norm of the normal component of the velocity) in the directions normal to the field, while the center of the orbit, called the guiding center, moves freely along a filed line. Keeping fixed the initial condition, the stronger the magnetic field the faster the rotation of the particle when compared with the drift along the filed direction and smaller the portion of space 


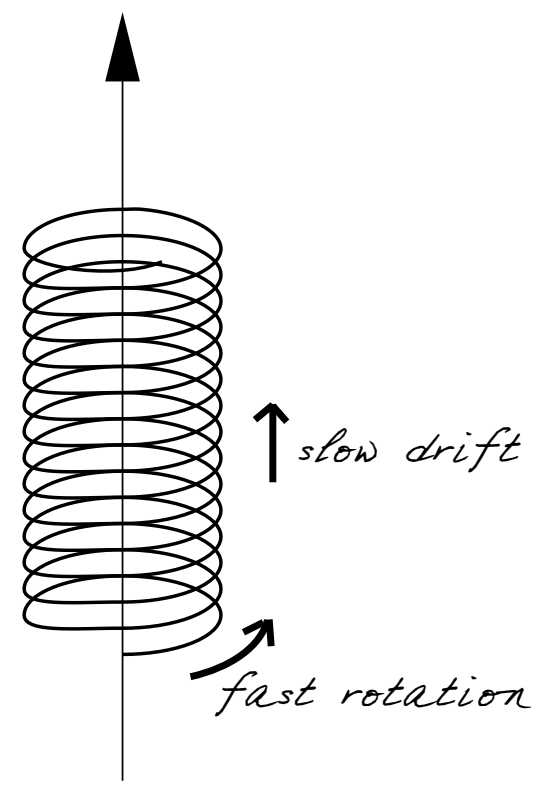

a)

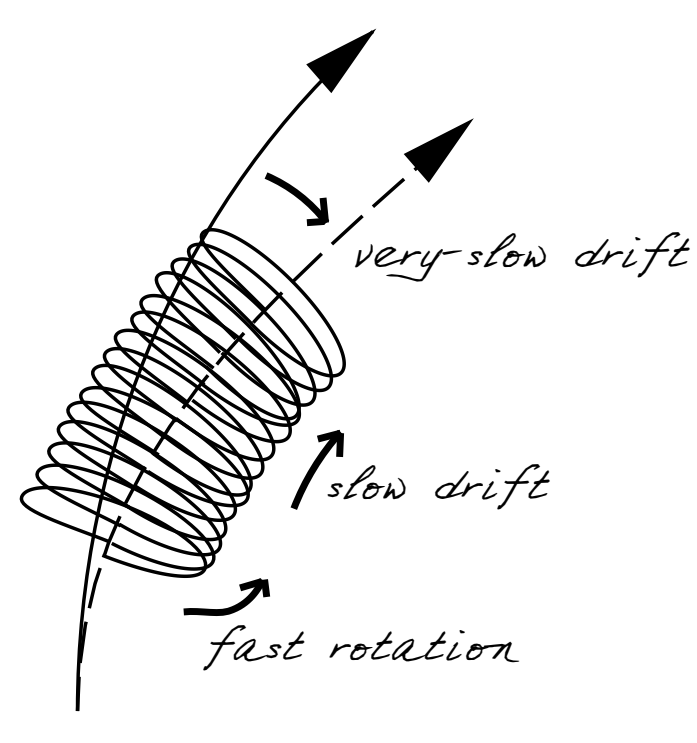

b)

Figure 1: Different time scale behaviours of a charged particle in a strong magnetic field: a) fast rotation of the particle and slow guiding center drift in a homogeneous field; $b$ ) in the inhomogeneous case the guiding center drifts away from the filed line very-slowly.

explored by the particle around the filed line. This indicates, to the one side, the presence of different time scales in the dynamics of the system and gives, on the other hand, the reason why the motion in a very strong magnetic filed may be studied along the same lines as that in a weakly inhomogeneous one. Let us introduce now a small inhomogeneity in the field. The picture of the motion should not change substantially. The particle, in fact, keeps on rotating around its guiding center, the frequency and the radius weakly depending now on the position, and the guiding center still drifts along a field line. In this case, however, the guiding center do not remains exactly on a single field line, it starts drifting very-slowly in the directions normal to the filed. Three different time scale behaviours of the system may therefore be distinguished: the fast rotation of the particle around the guiding center, the slow drift of the guiding center along a magnetic field line and the very-slow drift of the guiding center in the direction normal to the field. The situation is sketched in Fig. [1]b. The stronger the magnetic field the cleaner the separation 
among the three degrees of freedom.

An outlook to the canonical structure of the homogeneous case makes immediately clear how the introduction of kinematical momenta and guiding center operators allows to separate the three degrees of freedom of the system. This is briefly reported in section 2 where the relevant notations of the paper are also set up. After discussing the geometrical complications involved in the adiabatic motion of a charged particle in an inhomogeneous magnetic field, section 3, an appropriate set of non-canonical operators generalizing the one used in the discussion of the homogeneous problem is constructed in section 4 . These are obtained as formal power series in the magnetic length $l_{B}=\sqrt{\hbar c / e B}$ which appears naturally as the adiabatic parameter of the theory. The Pauli Hamiltonian describing the motion of the particle is then rewritten in terms of the new adiabatic operators in sections 5 and 6 , whereas the anholonomic effects appearing in the adiabatic separation of the fast and slow/very-slow degrees of freedom are discussed in section 7 . Our results are summarized in equations (42), (43) and (44). In the classical limit these reproduce correctly the classical theory. Section 8 contains our conclusions.

\section{Canonical structure of the guiding center motion}

Magnetic interactions appearing essentially as modifications of the canonical structure of a dynamical system it is worthwhile to start by briefly discussing this peculiarity in the elementary case of a quantum charged spinning particle in a homogeneous magnetic filed. This allows us to immediately focus on the heart of the problem establishing at the same time terminology and notations. We consider therefore a spin- $1 / 2$ particle of mass $m$, charge $e$ and gyromagnetic factor $g$ moving in space under the influence of the homogeneous filed $\boldsymbol{B}(\vec{x})=B \hat{\boldsymbol{z}}$. As in the inhomogeneous case, to be discussed later on, the physical dimension of the magnetic field is reabsorbed in the scale factor $B$, the inverse square root of which, opportunely rescaled, will play the role of the adiabatic parameter of our theory. Introducing an arbitrary choice of the vector potential $\boldsymbol{a}$ for the dimensionless filed $\boldsymbol{B}(\vec{x}) / B, \operatorname{rot} \boldsymbol{a}=\hat{\boldsymbol{z}}$, the motion of the particle is described by the Pauli Hamiltonian

$$
\mathcal{H}=\frac{1}{2 m}\left(-i \hbar \nabla-\frac{e B}{c} \boldsymbol{a}\right)^{2}+g \frac{\hbar e B}{m c} \hat{\boldsymbol{z}} \cdot \boldsymbol{\sigma}
$$

$\boldsymbol{\nabla}=\left(\partial_{x}, \partial_{y}, \partial_{z}\right)$ denoting the gradient operator and $\boldsymbol{\sigma}=\left(\sigma_{x}, \sigma_{y}, \sigma_{z}\right)$ the matrix-valued vector constructed by means of the three Pauli matrices. As well known the solution of this simple problem was first obtained by Landau at the beginning of the thirties and leads naturally to replace the standard set of canonical operators $p_{i}=-i \hbar \partial_{i}, x^{i}, i=1,2,3$, 
by the gauge invariant kinematical momenta $\pi_{i}=p_{i}-(e B / c) a_{i}$ and the guiding center operators $X^{i}=x^{i}+(c / e B) \varepsilon^{i j} \pi_{j}$. A very rapid computation yields the nonvanishing commutation relation among the new variables

$$
\left[\pi_{2}, \pi_{1}\right]=-i \frac{\hbar e B}{c}, \quad\left[\pi_{3}, X^{3}\right]=-i \hbar, \quad\left[X^{1}, X^{2}\right]=-i \frac{\hbar c}{e B},
$$

indicating $\pi_{2}-\pi_{1}, \pi_{3}-X^{3}$ and $X^{1}-X^{2}$ as couples of conjugates variables. Moreover, the scale dependence of the commutators (2) allows to identify the three couple of operators as describing respectively the fast, the slow and the very-slow degrees of freedom of the system (see eg. [6]). In terms of the new gauge invariant operators Hamiltonian (1) rewrites in the very simple form $\mathcal{H}=\left(\pi_{1}{ }^{2}+\pi_{2}{ }^{2}+\pi_{3}{ }^{2}\right) / 2 m+g \hbar e B \sigma_{3} / m c$. The harmonic oscillator term $\left(\pi_{1}{ }^{2}+\pi_{2}{ }^{2}\right) / 2 m$ takes into account the rapid rotation of the particle around its guiding center while the free term $\pi_{3}^{2} / 2 m$ the slow drift of the guiding center along the straight magnetic field lines. The very-slow variables $X^{1}$ and $X^{2}$ being constant of motion, the guiding center do not move in the directions normal to the field. Let us stress that in the canonical formalism the spatial rotation of the particle around its guiding center is taken into account by the phase space trajectory of a couple of conjugate variables: the particle's velocity components in the directions normal to the field: $\pi_{1}$ and $\pi_{2}$. The presence of an external magnetic field produces therefore a rotation of the canonical structure, mixing up spatial coordinates and canonical momenta in new canonical operators adapting to the different time scale behaviours of the particle! In section 4 we will construct such "adapted operators" - as power series in the adiabatic parameter - for the motion of a quantum charged spinning particle in an arbitrary magnetic filed. This allows to extend to quantum mechanics the Hamiltonian approach to the classical guiding center motion developed by R.G. Littlejohn [1]. The case of a magnetic filed with constant direction has been previously considered in $[\overline{6}]$. Before to proceed some preparatory material is however necessary.

First of all it is convenient to introduce dimensionless quantities by factorizing the energy scale $\hbar \omega_{B}, \omega_{B}=e B / m c$, from the Hamiltonian. This leads to redefine kinematical momenta and guiding center operators as

$$
\begin{aligned}
& \pi_{i}=-i l_{B} \partial_{i}-l_{B}{ }^{-1} a_{i}(\vec{x}) \\
& X^{i}=x^{i}+l_{B} \varepsilon^{i j} \pi_{j} .
\end{aligned}
$$

$l_{B}=\sqrt{\hbar c / e B}$ being the magnetic length. The relevant commutation relations may so be 
recast in the compact and very convenient form

$$
\begin{aligned}
& \left.\begin{array}{l}
{\left[\pi_{i}, \pi_{j}\right]=i \varepsilon_{i j}} \\
{\left[\sigma_{i}, \sigma_{j}\right]=i \varepsilon_{i j k} \sigma_{k}}
\end{array}\right\} \quad \text { fast } \\
& {\left[\pi_{i}, X^{j}\right]=-i l_{B} \delta_{i}^{3} \delta_{3}^{j} \quad \text { slow }} \\
& {\left[X^{i}, X^{j}\right]=-i l_{B}{ }^{2} \varepsilon^{i j} \quad \text { very-slow }}
\end{aligned}
$$

where the spin variables have also been considered.

As a second and more serious task the geometrical structure responsible for the anholonomic effects appearing in the adiabatic motion in a strong magnetic field has to be discussed.

\section{Magnetism and geometric-magnetism}

The beautiful analysis of the adiabatic separation of fast and slow degrees of freedom in a quantum system proposed by M.V. Berry [0], H. Kuratsuji \& S. Iida [8], J. Moody, A. Shapere \& F. Wilczek [9], R. Jackiw [10] and others, has pointed out that in lowest order the reaction of the fast to the slow dynamics is through a geometry-induced gauge structure resembling that of (electro-)magnetism. This phenomenon has been identified and found to be important in a variety of physical contexts [11] and has been recently referred by M.V. Berry \& J.M. Robbins as geometric-magnetism [12. A rather curious fact, first pointed out by R.G. Littlejohn in a series of beautiful papers on the canonical structure of classical guiding center motion [1], is that, in some circumstances, magnetism itself may generate geometric-magnetism. The aim of the present section is that of discussing the geometry involved in such "magnetic-induced geometric-magnetism".

For shake of clearness it is useful to begin by briefly recalling the geometrical character of the kinematical quantities characterizing the motion of a particle in space. This will led to a rather intuitive picture of the geometrical structure involved in the adiabatic motion of a charged spinning particle in a strong magnetic field, allowing, at the same time, to frame it in a general and rigorous context. As well known the state of a particle moving in space is completely characterized by its position $\vec{x}$ and its velocity $\vec{v}$, i.e. by a point in the tangent bundle $T R^{3}$ of the three-dimensional Euclidean space $R^{3}$. The flat parallel transport of $R^{3}$ makes it natural to parameterize every fiber $T_{\vec{x}} R^{3}$ of the bundle by means of a fixed reference frame in $R^{3}$, that is, to identify the tangent space in every point $\vec{x}$ with the physical space itself. Such an identification is certainly very useful in most circumstances, but it is a convention after all. In principle we are free to choose arbitrarily the frame of $T_{\vec{x}} R^{3}$ in every $\vec{x}$, the parallel transport — and not the way in which 


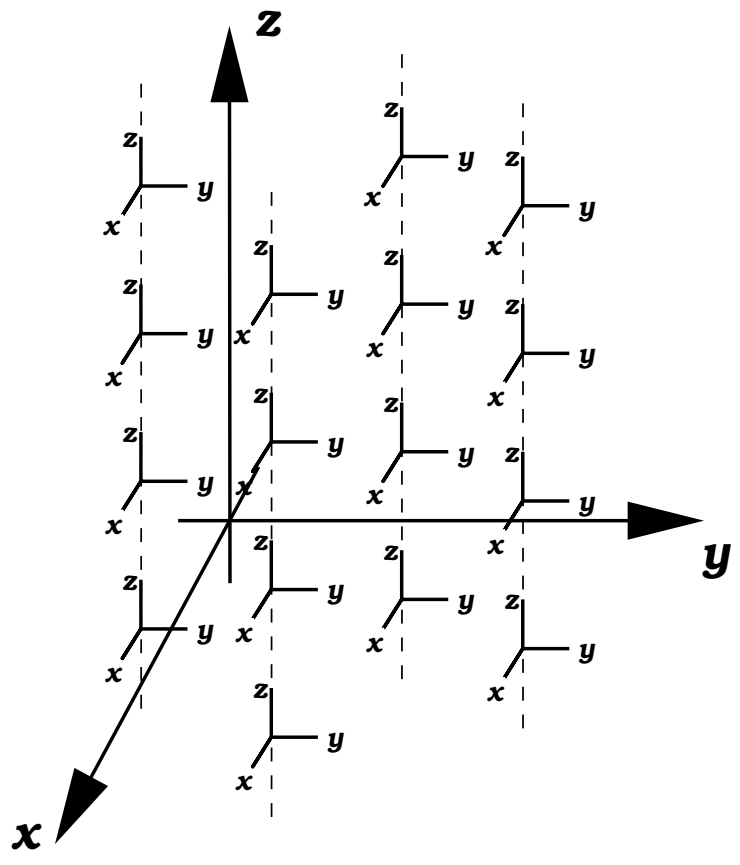

a)

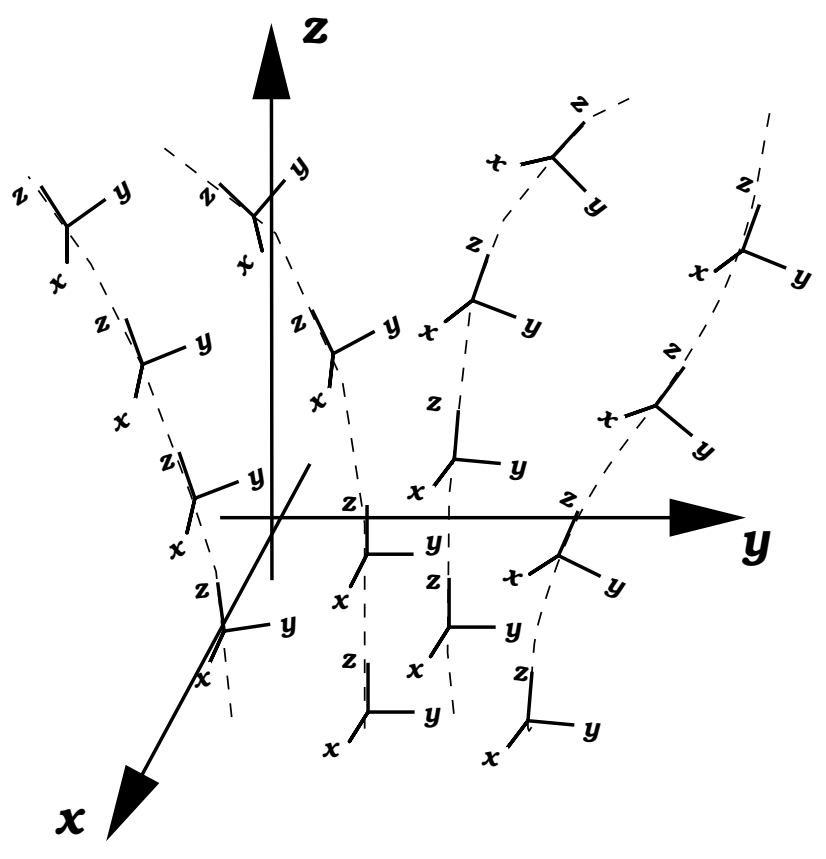

b)

Figure 2: Framing the tangent bundle $T R^{3}$ of the physical space: a) by means of a single fixed frame in $R^{3} ; b$ ) by using local reference frames adapting to the magnetic field lines geometry.

we describe it-being all that matters. This freedom of arbitrarily rotating the reference frame of the tangent space in every point $\vec{x}$, a local $\mathrm{SO}(3)$ symmetry, plays a crucial role in what follows. To visualize the situation, therefore, we shall picture the Euclidean space as filled up with orthonormal reference frames. To start with, we can imagine all them as combed parallel to a single fixed frame $\{\hat{\boldsymbol{x}}, \hat{\boldsymbol{y}}, \hat{\boldsymbol{z}}\}$ in $R^{3}$ (see Fig.2 2a), but even in a flat geometry, this is not always the better choice.

\section{The magnetic line bundle}

As qualitatively sketched above, the motion of a charged-spinning particle in a strong magnetic filed is characterized by the separation of time scales in the three degrees of freedom, making the system amenable to a perturbative analysis. In the lowest order 
approximation the particle performs a fast rotation in the plane normal to the field line at which its guiding center is located. This is taken into account by the two components normal to the field of the particle's velocity (to this order a couple of conjugate variables). Disregarding the slow drift of the guiding center along the filed line and the very-slow motion, therefore, the velocity of a particle which guiding center is located in $\vec{x}$ is effectively constrained to the plane $\boldsymbol{\mu}_{\vec{x}}$ generated by the vectors normal to the field in $\vec{x}$. In every point of the space the magnetic filed $\boldsymbol{b}(\vec{x})$ picks the complex line $\boldsymbol{\mu}_{\vec{x}}$ out of the tangent space $T_{\vec{x}} R^{3}$, reducing the tangent bundle $T R^{3}$ to a complex line bundle, hereafter the magnetic line bundle $\mathcal{M}$. It is then natural to use the local $S O(3)$ symmetry of the theory to adapt the parameterization of $T R^{3}$ to the subbundle $\mathcal{M}$ by combing, say, the $\hat{\boldsymbol{z}}$ direction of the frame of every $T_{\vec{x}} R^{3}$ according to the direction of the field. We so smoothly introduce point dependent adapted reference frames $\left\{\boldsymbol{e}_{1}, \boldsymbol{e}_{2}, \boldsymbol{e}_{3}\right\}$ in such a way that in every point $\boldsymbol{e}_{1}(\vec{x}), \boldsymbol{e}_{2}(\vec{x})$ parameterize $\boldsymbol{\mu}_{\vec{x}}$ while $\boldsymbol{e}_{3}(\vec{x})$ is aligned with $\boldsymbol{b}(\vec{x})$ (see Fig2b). Such reference frames are commonly used in the discussion of geometrically non trivial physical problems such as in general relativity and are referred as anholonomic frames. It is worthwhile to note that fixing $\boldsymbol{e}_{3}$ according to the filed direction reduces the local $S O(3)$ symmetry of $T R^{3}$ into the local $S O(2) \equiv U(1)$ symmetry of $\mathcal{M}$. The vectors $\boldsymbol{e}_{1}(\vec{x})$ and $\boldsymbol{e}_{2}(\vec{x})$ are in fact determined up to the rotation

$$
\begin{aligned}
& \boldsymbol{e}_{1}(\vec{x}) \rightarrow \boldsymbol{e}_{1}(\vec{x}) \cos \chi(\vec{x})-\boldsymbol{e}_{2}(\vec{x}) \sin \chi(\vec{x}) \\
& \boldsymbol{e}_{2}(\vec{x}) \rightarrow \boldsymbol{e}_{1}(\vec{x}) \sin \chi(\vec{x})+\boldsymbol{e}_{2}(\vec{x}) \cos \chi(\vec{x})
\end{aligned}
$$

$\chi(\vec{x})$ being a point dependent angle. This residual ambiguity will result in the gauge freedom of our theory.

\section{Magnetic line bundle geometry}

We may now wonder how the vectors lying in $\mathcal{M}$ are transported from point to point, that is, whether the geometry of the magnetic line bundle is trivial or not. To this task we proceed in two steps. Considering a vector $\boldsymbol{w}(\vec{x})=w^{\nu} \boldsymbol{e}_{\nu}(\vec{x}), \nu=1,2$, in $\boldsymbol{\mu}_{\vec{x}}$, we first transport it from the point $\vec{x}$ to the infinitesimally closest point $\vec{x}+d \vec{x}$ by means of the Euclidean parallel transport of $R^{3}$ and, second, we project it onto the plane $\boldsymbol{\mu}_{\vec{x}+d \vec{x}}$. (i) the Euclidean parallel transport of $\boldsymbol{w}$ in $\vec{x}+d \vec{x}$ may be immediately evaluated as

$$
\boldsymbol{w}(\vec{x}+d \vec{x})=\boldsymbol{w}(\vec{x})-w^{\nu}\left(\boldsymbol{e}_{\nu} \cdot \partial_{k} \boldsymbol{e}_{i}\right) d x^{k} \boldsymbol{e}_{i}
$$

\footnotetext{
${ }^{1}$ This subbundle of $T R^{3}$ may be identified with the plane bundle of B. Felsager \& J.M. Leinaas [13]. See also the related paper of F. Gliozzi [14].
} 
latin indices running over $1,2,3$, greek indices over 1,2 and where the sum over repeated indices is implied 2 . The three quantities $\boldsymbol{e}_{1} \cdot \partial_{k} \boldsymbol{e}_{2}, \boldsymbol{e}_{1} \cdot \partial_{k} \boldsymbol{e}_{3}$ and $\boldsymbol{e}_{2} \cdot \partial_{k} \boldsymbol{e}_{3}$ characterize the flat parallel transport of $R^{3}$ in the anholonomic frame (it is in fact possible to make them vanishing by rotating the adapted frames $\left\{\boldsymbol{e}_{1}, \boldsymbol{e}_{2}, \boldsymbol{e}_{3}\right\}$ back to fixed directions in every point). (ii) the projection onto $\boldsymbol{\mu}_{\vec{x}+d \vec{x}}$ yields then

$$
\left.\boldsymbol{w}(\vec{x}+d \vec{x})\right|_{\boldsymbol{\mu}}=\boldsymbol{w}(\vec{x})-w^{\mu}\left(\boldsymbol{e}_{1} \cdot \partial_{k} \boldsymbol{e}_{2}\right) d x^{k} \varepsilon_{\mu}{ }^{\nu} \boldsymbol{e}_{\nu},
$$

indicating that the parallel transport along the infinitesimal path connecting $\vec{x}$ to $\vec{x}+d \vec{x}$ produces the vector $\boldsymbol{w}$ to be rotated by the infinitesimal angle $d \alpha=\left(\boldsymbol{e}_{1} \cdot \partial_{k} \boldsymbol{e}_{2}\right) d x^{k}$. When parallel transported along a finite closed path $\gamma$ the vector will therefore return to the starting point rotated by the angle [13

$$
\alpha \boldsymbol{\gamma}=\oint_{\boldsymbol{\gamma}}\left(\boldsymbol{e}_{1} \cdot \partial_{k} \boldsymbol{e}_{2}\right) d x^{k}
$$

this quantity being in general different from zero, the geometry of the magnetic line bundle results not flat. The operation of locally projecting onto the plane $\boldsymbol{\mu}$ reduces the trivial $S O(3)$ local symmetry of the theory to a non-trivial $S O(2) \equiv U(1)$ local symmetry! This local structure is described by a magnetic-like $U(1)$ gauge theory. The parallel transport of the magnetic line bundle $\mathcal{M}$, results in fact completely characterized by the vector

$$
\mathcal{A}_{k}=\boldsymbol{e}_{1} \cdot \partial_{k} \boldsymbol{e}_{2}
$$

the connection one-form of $\mathcal{M}$. $\mathcal{A}$ appears in the theory as a geometry-induced vector potential (to not be confused with the vector potential $\boldsymbol{a}$ representing the real magnetic field $\boldsymbol{b})$. A point dependent redefinition of the local basis $\left\{\boldsymbol{e}_{1}(\vec{x}), \boldsymbol{e}_{2}(\vec{x})\right\}$ plays in fact the same role of a gauge transformation, the rotation (6) producing the vector (7) to transform according to $\mathcal{A}_{k} \rightarrow \mathcal{A}_{k}+\partial_{k} \chi$. The associate geometry-induced magnetic filed $\mathcal{B}_{k}=$ $\varepsilon_{k m n} \mathcal{B}_{m n}, \mathcal{B}_{m n}=\partial_{m} \mathcal{A}_{n}-\partial_{n} \mathcal{A}_{m}$ the curvature two-form of $\mathcal{M}$, may also be considered. It is obviously a gauge invariant quantity and, being the rotor of a vector field, satisfies the Bianchi identity $\operatorname{div} \mathcal{B}=0$.

While the geometry-induced vector potential $\mathcal{A}$ completely characterizes the intrinsic geometry of the magnetic line bundle $\mathcal{M}$, the other two quantities

$$
\begin{aligned}
& l_{1 k}=\boldsymbol{e}_{1} \cdot \partial_{k} \boldsymbol{e}_{3}, \\
& l_{2 k}=\boldsymbol{e}_{2} \cdot \partial_{k} \boldsymbol{e}_{3},
\end{aligned}
$$

\footnotetext{
${ }^{2}$ This notation will be employed throughout the rest of this paper.

${ }^{3}$ The vectors $\boldsymbol{e}_{1}, \boldsymbol{e}_{2}$ and $\boldsymbol{e}_{3}$ being orthonormal in every point $\vec{x}, \boldsymbol{e}_{i} \cdot \boldsymbol{e}_{j}=\delta_{i j}$, these are the only independent quantities.
} 
describing the flat parallel transport of $R^{3}$ in the anholonomic frame $\left\{\boldsymbol{e}_{1}, \boldsymbol{e}_{2}, \boldsymbol{e}_{3}\right\}$ may be seen a taking into account its extrinsic geometry. Since the curvature of the tangent bundle $T R^{3}$ is zero the three quantities $\mathcal{A}, \boldsymbol{l}_{\mathbf{1}}$ and $\boldsymbol{l}_{\mathbf{2}}$ are obviously not independent, being related by the equivalent of the Gauss, Codazzi-Mainardi and Ricci equations. The ladder, as an example, allows to re-express the geometry-induced gauge field $\mathcal{B}$ entirely in terms of $\boldsymbol{l}_{\mathbf{1}}$ and $\boldsymbol{l}_{\mathbf{2}}$ as

$$
\mathcal{B}=l_{1} \wedge l_{2}
$$

$\mathcal{B}_{k l}=\left(l_{1 k} l_{2 l}-l_{1 l} l_{2 k}\right) / 2, \wedge$ indicating the external product of $R^{3}$ [13]. With respect to the point dependent rotation (6) $\boldsymbol{l}_{\mathbf{1}}$ and $\boldsymbol{l}_{\mathbf{2}}$ transform as vectors $\left(\boldsymbol{l}_{\mathbf{1}} \rightarrow \boldsymbol{l}_{\mathbf{1}} \cos \chi-\boldsymbol{l}_{\mathbf{2}} \sin \chi\right.$, $\left.\boldsymbol{l}_{\mathbf{2}} \rightarrow \boldsymbol{l}_{\mathbf{1}} \sin \chi+\boldsymbol{l}_{\mathbf{2}} \cos \chi\right)$ making the gauge invariance of $\mathcal{B}$ manifest.

\section{Magnetic filed lines geometry}

Thought the geometry of a magnetic filed is completely characterized by two independent function (e.g. the two independent components of the real magnetic field $\boldsymbol{b}$, or of the geometry-induced magnetic field $\mathcal{B}$, etc. ) it may be useful to look at the problem from different points of view. We may wonder, as an example, how the intrinsic/extrinsic geometry of the line bundle $\mathcal{M}$ is related to the geometry of magnetic filed lines. To this task we start by observing that the projection along the field direction of the two vectors $\boldsymbol{l}_{\mathbf{1}}, \boldsymbol{l}_{\mathbf{2}}$ may be identified with the two second fundamental forms of the embedding of the magnetic field lines in $R^{3}$ [15]. In every point of the space the curvature $k$ of the magnetic filed line going through that point may so be expressed as

$$
k=\sqrt{\left(\boldsymbol{e}_{3} \cdot \boldsymbol{l}_{\mathbf{1}}\right)^{2}+\left(\boldsymbol{e}_{3} \cdot \boldsymbol{l}_{\mathbf{2}}\right)^{2}} .
$$

In a similar way the projection along the filed direction of the geometry-induced vector potential $\mathcal{A}$ have to be identified with the normal fundamental form of the embedding of the field lines in $R^{3}$ (i.e. with the connection form induced by the Euclidean geometry onto the normal bundle of every filed line) [15]. Up to the gradient of an arbitrary function, representing again the freedom of arbitrarily rotating the reference frame in the normal planes, in every point of the space the torsion $\tau$ of the magnetic field line going through that point may be written as

$$
\tau=e_{3} \cdot \mathcal{A}
$$

Curvature and torsion completely characterize the geometry of every single magnetic filed line and contain, in principle, all the informations relative to the geometry of our problem. 
On the other hand we may also wonder about the global properties of the foliation of $R^{3}$ in terms of field lines. Of particular relevance for the adiabatic motion of a particle in an external magnetic field is the possibility of foliating space by means of surfaces everywhere orthogonal to the field lines. By virtue of Frobenius theorem this is controlled by the vanishing of the scalar $\mathcal{F}=\boldsymbol{e}_{3} \cdot \operatorname{rot} \boldsymbol{e}_{3}$. In terms of the magnetic line bundle geometry

$$
\mathcal{F}=e_{1} \cdot l_{2}-e_{2} \cdot l_{1}
$$

The magnetic filed lines torsion $\tau$ and the Frobenius invariant $\mathcal{F}$ play a crucial role in the description of the anholonomic effects appearing in the adiabatic motion of a charged particle in a strong magnetic field.

\section{Adiabatic quantum variables}

We are now ready for the construction of a set of adiabatic operators adapting to the different time scale behaviours of a quantum particle in a strong, but otherwise arbitrary, magnetic field. Let us consider therefore a spin-1/2 particle of mass $m$, charge $e$ and gyromagnetic factor $g$ moving in space under the influence of the inhomogeneous magnetic field $\boldsymbol{B}(\vec{x})=B \boldsymbol{b}(\vec{x})$, the physical dimension of the filed being again reabsorbed in the scale factor $B$. Denoting by $\boldsymbol{a}$ an arbitrary choice of the vector potential, rot $\boldsymbol{a}=\boldsymbol{b}$, the dynamics of the system is described by the Pauli Hamiltonian

$$
\mathcal{H} / \hbar \omega_{B}=\frac{1}{2} \pi_{i} \pi_{i}+g b_{i}(\vec{x}) \sigma_{i}
$$

where the kinematical momenta $\pi_{i}=-i l_{B} \partial_{i}-a_{i}(\vec{x}) / l_{B}$ have been introduced. The inhomogeneity of the magnetic filed makes Hamiltonian (13) to depend on the position operators $\vec{x}$, explicitly through spin term $g b_{i}(\vec{x}) \sigma_{i}$ and implicitly through the commutation relations of the $\pi_{i} \mathrm{~s}$. In spite of the simple quadratic dependence of (13) on the kinematical momenta, $\pi_{1}$ and $\pi_{2}$ are in fact no longer conjugate variables and neither commute with $\pi_{3}$ : the set of operators $\left\{\pi_{i}, x^{i} ; i=1,2,3\right\}$ fulfil the commutation relations

$$
\left[\pi_{i}, \pi_{j}\right]=i b_{i j}(\vec{x}), \quad\left[\pi_{i}, x^{j}\right]=-i l_{B} \delta_{i}^{j}, \quad\left[x^{i}, x^{j}\right]=0,
$$

$b_{i j}(\vec{x})=\varepsilon_{i j k} b_{k}(\vec{x})$ denoting the skew-symmetric two-form associated to the field. In the lowest approximation we nevertheless expect the relevant degree of freedom of the system to be taken into account by the two components of the particle's velocity normal to 
the filed. Considering the position operators $x^{i}$ s as adiabatic parameters driving the fast motion of the system we expect therefore the rapid rotation of the particle around its guiding center to be separated from the slow and very-slow motion by simply referring the kinematical momenta to the adapted frames introduced in the previous section. For the shake of concreteness we shall indicate by $R_{i}{ }^{j}(\vec{x})$ the point dependent rotation bringing the fixed frame $\{\hat{\boldsymbol{x}}, \hat{\boldsymbol{y}}, \hat{\boldsymbol{z}}\}$ into the adapted frame $\left\{\boldsymbol{e}_{1}(\vec{x}), \boldsymbol{e}_{2}(\vec{x}), \boldsymbol{e}_{3}(\vec{x})\right\}$. This allows to decompose the field $\boldsymbol{b}(\vec{x})$ in terms of its norm $b=\sqrt{\boldsymbol{b} \cdot \boldsymbol{b}}$ and its direction $\boldsymbol{b} / b=R_{i}{ }^{j} \hat{\boldsymbol{z}}_{j}$ as $b_{i}(\vec{x})=b(\vec{x}) R_{i}{ }^{j}(\vec{x}) \hat{\boldsymbol{z}}_{j}$. Once the rotation has been performed the kinematical momentum along the field direction decouples, up to higher order terms in the adiabatic parameter $l_{B}$, from the other two components. The commutator of these, on the other hand, results to be proportional to $b(\vec{x})$. Stated in a different way, in the adapted frame the particle sees an effective magnetic filed of constant direction and intensity $b(\vec{x})$. To make the velocity components normal to the filed in a couple of conjugate operators it is now sufficient to rescale them by the point dependent factor $b^{-1 / 2}(\vec{x})$ (see [6]). We shall indicate by $D_{i}{ }^{j}(\vec{x})$ the point dependent dilatation $D_{i}{ }^{j}=\operatorname{diag}\left(b^{1 / 2}, b^{1 / 2}, 1\right)$ rescaling the first and second components of a vector by $b^{1 / 2}$ and letting the third one unchanged.

In order to construct operators adapting to the fast time scale behaviour of the system two point dependent operations have therefore to be performed: (i) a rotation $R_{i}{ }^{j}(\vec{x})$ to the local adapted frame and (ii) a dilatation $D_{i}{ }^{j}(\vec{x})$ rescaling the normal components of the kinematical momenta. The particle coordinates being not external parameters but dynamical variables of the problem these operations will produce higher order corrections in the various commutators. We shall therefore proceed order by order in the adiabatic parameter $l_{B}$ by constructing sets of adiabatic operators fulfilling the desired commutation relation up to a given order in $l_{B}$ : at the $n$-th order we shall look for a set of operators $\left\{\Pi_{i}^{(n)}, X_{(n)}^{i} ; i=1,2,3\right\}$ fulfilling the conditions

- $\Pi_{1}^{(n)}, \Pi_{2}^{(n)}$ are a couple of conjugate operators up to terms of order $l_{B}{ }^{n}$,

- $\Pi_{3}^{(n)}, X_{(n)}^{1}, X_{(n)}^{2}, X_{(n)}^{3}$ commute with $\Pi_{1}^{(n)}, \Pi_{2}^{(n)}$ up to terms of order $l_{B}{ }^{n}$,

- in the limit of a homogeneous filed, $\boldsymbol{b}(\vec{x}) \rightarrow \hat{\boldsymbol{z}}$, the adiabatic kinematical momenta $\Pi_{i}^{(n)}$ s and guiding center operators $X_{(n)}^{i}$ s should reduce to the expressions (3) and (四) respectively.

Our present task being that of separating the fast degree of freedom from the slow and very-slow motion, we do not insist for the moment $X_{(n)}^{3}-\Pi_{3}^{(n)}$ and $X_{(n)}^{1}-X_{(n)}^{2}$ to be couple of conjugate operators as in the homogeneous case. 
For computational proposes it is very convenient to use a compact notation which does not distinguish among the physical dislike directions along and normal to the field. This probably obscures a while the physical contents of the various expression but greatly simplifies formal manipulations. When necessary we will therefore expand the notation in order to shad light on the physics involved. For the moment we proceed in the opposite direction by introducing the point dependent matrix

$$
\beta_{i}{ }^{j}(\vec{x})=D_{i}^{-1 k}(\vec{x}) R_{k}^{-1 j}(\vec{x})
$$

representing the successive application of the two operations necessary to construct the adapted kinematical momenta in the lowest order. This allows to rewrite the skewsymmetric two-form $b_{i j}(\vec{x})$ in terms of $\varepsilon_{k l}=\varepsilon_{k l 3}$ (representing a homogeneous filed directed along $\hat{\boldsymbol{z}})$

$$
b_{i j}(\vec{x})=\beta_{i}^{-1 k}(\vec{x}) \beta_{j}^{-1 l}(\vec{x}) \varepsilon_{k l} .
$$

The matrix $\beta_{i}{ }^{j}$ and this representation of the filed result to be very useful in the construction of the adiabatic quantum variables.

\section{Zero-order operators}

In order to construct the zero-order operators fulfilling the desired conditions up to terms of order $l_{B}$ it is sufficient to operate the rotation and the dilatation discussed above

$$
\Pi_{i}^{(0)}=\frac{1}{2}\left\{\beta_{i}{ }^{k}, \pi_{k}\right\}
$$

the matrix $\beta_{i}{ }^{k}$ being evaluated in $\vec{X}_{(0)} \equiv \vec{x}$. The anticommutator $\{$,$\} is obviously intro-$ duced in order to make the $\Pi_{i}^{(0)}$ s Hermitian. A rapid computation confirms our deductions yielding the commutation relations fulfilled by the zero-order adiabatic operators as

$$
\begin{aligned}
& {\left[\Pi_{i}^{(0)}, \Pi_{j}^{(0)}\right]=i \varepsilon_{i j}-i \frac{l_{B}}{2} \varepsilon_{i j h} \varepsilon^{h k l}\left\{\beta_{k}{ }^{m} \Gamma_{m l}{ }^{n}, \Pi_{n}^{(0)}\right\}} \\
& {\left[\Pi_{i}^{(0)}, X_{(0)}^{j}\right]=-i l_{B} \beta_{i}{ }^{j}} \\
& {\left[X_{(0)}^{i}, X_{(0)}^{j}\right]=0}
\end{aligned}
$$

where $\Gamma_{k i}^{j}=\left(\partial_{k} \beta_{i}{ }^{h}\right) \beta_{h}^{-1 j}$ and all the functions are evaluated in $\vec{X}_{(0)} \cdot \Pi_{1}^{(0)}$ and $\Pi_{2}^{(0)}$ are conjugate operators up to $\mathcal{O}\left(l_{B}\right)$. The commutators depend on the derivative of magnetic 
filed through the vector-valued matrix

$$
\left(\Gamma_{k}\right)_{i}{ }^{j}=\left(\begin{array}{ccc}
-\frac{1}{2} \frac{\partial_{k} b}{b} & -\mathcal{A}_{k} & -b^{-1 / 2} l_{1 k} \\
\mathcal{A}_{k} & -\frac{1}{2} \frac{\partial_{k} b}{b} & -b^{-1 / 2} l_{2 k} \\
b^{1 / 2} l_{1 k} & b^{1 / 2} l_{2 k} & 0
\end{array}\right)
$$

allowing to clearly distinguish the effects produced by a variation of norm of the magnetic filed from that produced by a change of direction. The ladder are entirely geometrical in character being taken into account by the magnetic line bundle connection form $\mathcal{A}$ and by the two extrinsic vectors $\boldsymbol{l}_{\mathbf{1}}$ and $\boldsymbol{l}_{\mathbf{2}}$.

\section{First-order operators}

Whereas the construction of the zero-order operators is in some way suggested by the physics of the problem, a more technical effort is required for higher order terms. The form of the first-oder guiding center operators is nevertheless suggested by the corresponding homogeneous expression (4),

$$
X_{(1)}^{i}=X_{(0)}^{i}+\frac{l_{B}}{2} \varepsilon^{k l}\left\{\beta_{k}^{i}, \Pi_{l}^{(0)}\right\}
$$

the matrix $\beta_{k}{ }^{i}$ being again evaluated in $\vec{X}_{(0)}$. We immediately obtain the new commutation relation

$$
\begin{aligned}
& {\left[\Pi_{i}^{(0)}, \Pi_{j}^{(0)}\right]=i \varepsilon_{i j}-i \frac{l_{B}}{2} \varepsilon_{i j h} \varepsilon^{h k l}\left\{\beta_{k}{ }^{m} \Gamma_{m l}^{n}, \Pi_{n}^{(0)}\right\}+\mathcal{O}\left(l_{B}{ }^{2}\right),} \\
& {\left[\Pi_{i}^{(0)}, X_{(1)}^{j}\right]=-i l_{B} \delta_{i}^{3} \beta_{3}{ }^{j}+\mathcal{O}\left(l_{B}{ }^{2}\right),} \\
& {\left[X_{(1)}^{i}, X_{(1)}^{j}\right]=-i l_{B}{ }^{2} \varepsilon^{k l} \beta_{k}{ }^{i} \beta_{l}^{j}+\mathcal{O}\left(l_{B}{ }^{3}\right),}
\end{aligned}
$$

indicating the $\mathcal{O}\left(l_{B}{ }^{2}\right)$ decoupling of the adiabatic guiding center operators from $\Pi_{1}^{(0)}$ and $\Pi_{2}^{(0)}$. All the functions are now evaluated in $\vec{X}_{(1)}$. Though our analysis will be carried out up to $\mathcal{O}\left(l_{B}{ }^{2}\right)$, we also wrote the the first nonvanishing contribution to the commutators among the $X_{(1)}^{i} \mathrm{~s}$, which is of order $l_{B}{ }^{2}$. Even if unimportant in the present calculation, this allows us to visualize the very-slow time scale of the system.

The construction of the first-order kinematical momenta is performed by looking for order $l_{B}$ counterterms to be added to the $\Pi_{i}^{(0)}$ 's. These should be homogeneous second 
order polynomial in the $\Pi_{i}^{(0)}$ 's with coefficients depending on $\vec{X}_{(1)}$. A rather tedious computation produces

$$
\Pi_{i}^{(1)}=\Pi_{i}^{(0)}+l_{B} c_{i j}^{k l m n}\left\{\beta_{m}{ }^{h} \Gamma_{h n}^{j},\left\{\Pi_{k}^{(0)}, \Pi_{l}^{(0)}\right\}\right\},
$$

where $c_{i j}^{k l m n}=\frac{1}{24} \varepsilon_{i h} \varepsilon^{k h}\left(2 \delta_{j}^{l}+\delta_{j}^{3} \delta_{3}^{l}\right) \varepsilon^{m n}+\frac{1}{8} \delta_{i}^{3} \varepsilon^{k h}\left(\delta_{j}^{l}+\delta_{j}^{3} \delta_{3}^{l}\right) \varepsilon_{h g} \varepsilon^{g m n}$ and all the functions are evaluated in $\vec{X}_{(1)}$. When expanded these expressions do not look so complicated as a first sight. We nevertheless insist in keeping this notation which greatly simplifies the following manipulations. The commutation relations among the first-order adiabatic variables are obtained as

$$
\begin{aligned}
& {\left[\Pi_{i}^{(1)}, \Pi_{j}^{(1)}\right]=i \varepsilon_{i j}-i \frac{l_{B}}{4} \varepsilon_{i j k} \varepsilon^{k l}\left\{\frac{\operatorname{div} \boldsymbol{b}}{b}, \Pi_{l}^{(1)}\right\}+\mathcal{O}\left(l_{B}{ }^{2}\right),} \\
& {\left[\Pi_{i}^{(1)}, X_{(1)}^{j}\right]=-i l_{B} \delta_{i}{ }^{3} \beta_{3}{ }^{j}+\mathcal{O}\left(l_{B}{ }^{2}\right),} \\
& {\left[X_{(1)}^{i}, X_{(1)}^{j}\right]=-i l_{B}{ }^{2} \varepsilon^{k l} \beta_{k}{ }^{i} \beta_{l}{ }^{j}+\mathcal{O}\left(l_{B}{ }^{3}\right) .}
\end{aligned}
$$

It is very interesting to observe that a monopole singularity, that is a point of nonvanishing divergence, represents an obstruction in the construction of the adiabatic operators. Being concerned with real magnetic filed we nevertheless assume $\operatorname{div} \boldsymbol{b}=0$ and carry on in our adiabatic analysis. $\Pi_{1}^{(1)}$ and $\Pi_{2}^{(1)}$ are then conjugate operators commuting with all the remaining variables up to terms of order $l_{B}{ }^{2}$ and the fast degree of freedom decouples from the slow and very-slow motion up to terms of this order.

\section{A non-canonical set of operators}

At least in principle it is possible to repeat this construction an arbitrary number of times getting, as power series in $l_{B}$, a set of adiabatic non-canonical operators $\left\{\Pi_{i}, X^{i} ; i=\right.$ $1,2,3\}$ fulfilling the commutation relations

$$
\left[\Pi_{i}, \Pi_{j}\right]=i \varepsilon_{i j}, \quad\left[\Pi_{i}, X^{j}\right]=-i l_{B} \delta_{i}^{3} R_{3}^{-1 j}, \quad\left[X^{i}, X^{j}\right]=-i l_{B}^{2} \varepsilon^{k l} b^{-1} R_{k}^{-1 i} R_{l}^{-1 j},
$$

all the functions being now evaluated in $\vec{X}$. These formal series are in general-and have to be [16] - not convergent, representing anyway a very useful tool in the discussion of the adiabatic behaviour of the system. The description of the problem to a given order $n$ in the adiabatic parameter $l_{B}$ requires the knowledge of the first $n+1$ terms of the adiabatic series, so that up to terms of order $l_{B}{ }^{2}$ we may identify the $\Pi_{i} \mathrm{~s}$ and $X^{i} \mathrm{~s}$ with the $\Pi_{i}^{(1)} \mathrm{s}$ and $X_{(1)}^{i}$ s respectively. An outlook to the commutation relation (24) allows to clearly 
identify the dependence of the canonical structure on the variation of norm and direction of the magnetic filed. Whereas a suitable redefinition of reference frames in $T R^{3}$ allows to separate the fast degree of freedom from the others, the very-slow variables are made into a couple of non-conjugate operators by an inhomogeneous intensity while a variation of the filed direction even produces the mixing of very-slow and slow variables. The description of these by means of couples of conjugate operators requires the introduction of curvilinear coordinates in space [17], the so called Euler potentials [18]. We do not insist further on this point for the moment observing that under the action of $\Pi_{1}, \Pi_{2}$ and $\Pi_{3}$, $X^{1}, X^{2}, X^{3}$ the Hilbert space of the system separates in the direct sum of two subspaces describing respectively the rapid rotation of the particle and the guiding center motion.

\section{$5 \quad$ Expanding the Hamiltonian}

The adiabatic operators $\vec{\Pi}$ and $\vec{X}$ constructed in the previous section have been introduced in such a way to embody the expected features of the motion of a quantum charged particle in a weakly-inhomogeneous magnetic field. Their main advantage lies, in fact, in the very suitable form assumed by the Pauli Hamiltonian when rewritten in terms of them. To this task we have first to invert the power series expressing $\Pi_{i}$ and $X^{i}$ in terms of the operators $\pi_{i}$ s and $x^{i}$ s and, second, to replace these in (13). This yields the Hamiltonian as a power series in the magnetic length $l_{B}$,

$$
\mathcal{H}=\mathcal{H}^{(0)}+l_{B} \mathcal{H}^{(1)}+l_{B}{ }^{2} \mathcal{H}^{(2)}+\ldots
$$

allowing the adiabatic separation of the fast degree of freedom from the slow/very-slow motion and the evaluation of approximate expressions of the spectrum and of the wave functions of the system. In order to get the $\pi_{i} \mathrm{~s}$ and $x^{i} \mathrm{~s}$ in terms of the $\Pi_{i} \mathrm{~s}$ and $X^{i} \mathrm{~s}$ we first recall that $X^{i}=X_{(1)}^{i}+\mathcal{O}\left(l_{B}{ }^{2}\right)$. By rewriting $X_{(1)}^{i}$ in terms of the $\Pi_{i}^{(0)} \mathrm{s}$ and $X_{(0)}^{i}=x^{i} \mathrm{~s}$, (20), $\Pi_{i}^{(0)}$ in terms of the $\pi_{i} \mathrm{~s}$ and $x^{i} \mathrm{~s}$, (17), and by solving with respect to $x^{i}$, we then obtain $x^{i}$ as a function of the $\pi_{i}$ s and the $X^{i} \mathrm{~s}, x^{i}=x^{i}(\vec{\pi}, \vec{X})$. This allows to rewrite $\Pi_{i}^{(0)}$ as a function of the $\pi_{i}$ s and $X^{i}$ s. Recalling finally that $\Pi_{i}=\Pi_{i}^{(1)}+\mathcal{O}\left(l_{B}{ }^{2}\right)$ and using (22) we immediately get $\Pi_{i}$ in terms of the $\pi_{i}$ s and $X^{i} \mathrm{~s}, \Pi_{i}=\Pi_{i}(\vec{\pi}, \vec{X})$. The inversion of this relation, order by order in $l_{B}$, allows to get $\pi_{i}$ and $x^{i}$ in terms of the adiabatic operators. The computation gives

$$
\begin{aligned}
\pi_{i} & =\frac{1}{2}\left\{\beta^{-1}{ }_{i}^{j}, \Pi_{j}\right\}+\frac{l_{B}}{2} c_{j h}^{k l m n}\left\{\beta_{i}^{-1}{ }_{i} \beta_{m}^{o} \Gamma_{o n}{ }^{h},\left\{\Pi_{k}, \Pi_{l}\right\}\right\}+\mathcal{O}\left(l_{B}{ }^{2}\right), \\
x^{i} & =X^{i}-l_{B} \varepsilon^{k l} \beta_{k}^{i} \Pi_{l}+\mathcal{O}\left(l_{B}{ }^{2}\right),
\end{aligned}
$$


where $\mathrm{c}_{i j}^{k l m n}=\frac{1}{2} \delta_{i}^{n} \delta_{j}^{k} \varepsilon^{m l}-2 c_{i j}^{k l m n}$. As a useful check the commutation relations (14) may be reobtained by means of the (24).

The substitution of (26), (27) in the Pauli Hamiltonian (13) yields immediately the first two terms of the adiabatic expansion (25),

$$
\begin{aligned}
& \mathcal{H}^{(0)} / \hbar \omega_{B}=\frac{1}{2} \beta_{i}^{-1}{ }_{i} \beta^{-1}{ }_{i}^{l} \Pi_{k} \Pi_{l}+g b_{i} \sigma_{i} \\
& \mathcal{H}^{(1)} / \hbar \omega_{B}=\beta_{i}^{-1}{ }_{i}{ }^{-1}{ }_{i}^{q} \tilde{c}_{p j}^{k l m n} \beta_{m}^{o} \Gamma_{\text {on }}^{j}\left\{\Pi_{k} \Pi_{q} \Pi_{l}\right\}-g \varepsilon^{k l} \beta_{k}^{h}\left(\partial_{h} b_{i}\right) \sigma_{i} \Pi_{l}
\end{aligned}
$$

where the notation $\left\{\Pi_{k} \Pi_{q} \Pi_{l}\right\}=\Pi_{k} \Pi_{q} \Pi_{l}+\Pi_{l} \Pi_{q} \Pi_{k}$ has been introduced. In order to get some more physical insight in this expressions we now abandon our compact notation in favour of a more transparent one. By recalling the definition (15) of $\beta_{i}{ }^{j}(\vec{x})$, (19) of and $\Gamma_{i j}{ }^{k}(\vec{x})$ and the explicit expression of the inhomogeneous dilatation $D_{i}{ }^{j}(\vec{x})=$ $\operatorname{diag}\left(b^{1 / 2}(\vec{x}), b^{1 / 2}(\vec{x}), 1\right)$, we rewrite everything in terms of the magnetic field and of other quantities capable of a direct physical interpretation. The full expansion of the zero order Hamiltonian (28) gives

$$
\mathcal{H}^{(0)} / \hbar \omega_{B}=\frac{1}{2} \Pi_{3}^{2}+b\left[J+g\left(\boldsymbol{e}_{3} \cdot \boldsymbol{\sigma}\right)\right]
$$

where $J$ represents the harmonic oscillator Hamiltonian constructed by means of the canonical variables $\Pi_{1}$ and $\Pi_{2}, J=\left(\Pi_{1}^{2}+\Pi_{2}^{2}\right) / 2$, and the norm of the magnetic field $b(\vec{X})$ is evaluated in the adiabatic guiding center operators $\vec{X}$. We observe that while the $\Pi_{1}-\Pi_{2}$ degree of freedom decouples to this order from the slow and very-slow variables the spin does not. The separation, up to higher order terms, of the fast motion (rotation + spin) requires in fact a subsidiary zero order transformation which we will perform in the next section. For the moment let us observe that, up to the spin term, the zero order Hamiltonian (30) precisely embodies the expected behaviour of the system: the canonical couple of operators $\Pi_{1}-\Pi_{2}$ takes into account the fast rotation of the particle around its guiding center, while the non-canonical variables $\Pi_{3}-X^{3}$ describe the slow motion along the magnetic field lines by means of an effective "kinetic energy + potential energy" Hamiltonian. The norm of the magnetic filed $b(\vec{X})$ plays the role of the effective potential. As long as $\mathcal{O}\left(l_{B}{ }^{2}\right)$ terms are ignored the very-slow dynamical variables $X^{1}-X^{2}$ appear only as adiabatic parameters driving the slow motion, whereas a more accurate analysis indicates them as taking into account the very-slow drift in the directions normal to the field [6]. 
More complicated appears the full expression of the first order Hamiltonian (29). The replacement of $\beta_{i}{ }^{j}(\vec{x})$ and $\Gamma_{i j}{ }^{k}(\vec{x})$ by means of (15) and (19) yields in fact the expression

$$
\begin{aligned}
& \mathcal{H}^{(1)} / \hbar \omega_{B}=-b^{-1 / 2} \varepsilon^{\mu \nu}\left(\boldsymbol{e}_{\mu} \cdot \boldsymbol{\nabla} b\right)\left[\frac{2}{3} J_{\nu}+g\left(\boldsymbol{e}_{3} \cdot \boldsymbol{\sigma}\right) \Pi_{\nu}\right]-\frac{2}{3} b^{1 / 2}\left(\boldsymbol{e}_{\mu} \cdot \mathcal{A}\right) J_{\mu} \\
+ & {\left[\frac{1}{2}\left(\boldsymbol{e}_{1} \cdot \boldsymbol{l}_{\mathbf{2}}-\boldsymbol{e}_{2} \cdot \boldsymbol{l}_{\mathbf{1}}\right)-\left(\boldsymbol{e}_{\mathbf{3}} \cdot \boldsymbol{A}\right)\right] J \Pi_{3}+\frac{1}{4}\left(\boldsymbol{e}_{1} \cdot \boldsymbol{l}_{\mathbf{2}}+\boldsymbol{e}_{2} \cdot \boldsymbol{l}_{\mathbf{1}}\right)\left(\Pi_{1}{ }^{2}-\Pi_{2}{ }^{2}\right) \Pi_{3} } \\
- & \frac{1}{4}\left(\boldsymbol{e}_{1} \cdot \boldsymbol{l}_{\mathbf{1}}-\boldsymbol{e}_{2} \cdot \boldsymbol{l}_{\mathbf{2}}\right)\left\{\Pi_{1}, \Pi_{2}\right\} \Pi_{3}+b^{-1 / 2}\left[\left(\boldsymbol{e}_{3} \cdot \boldsymbol{l}_{\mathbf{2}}\right) \Pi_{1}-\left(\boldsymbol{e}_{3} \cdot \boldsymbol{l}_{\mathbf{1}}\right) \Pi_{2}\right] \Pi_{3}{ }^{2} \\
- & g b^{1 / 2} \varepsilon^{\mu \nu}\left[\left(\boldsymbol{e}_{\mu} \cdot \boldsymbol{l}_{\mathbf{1}}\right)\left(\boldsymbol{e}_{1} \cdot \boldsymbol{\sigma}\right)+\left(\boldsymbol{e}_{\mu} \cdot \boldsymbol{l}_{\mathbf{2}}\right)\left(\boldsymbol{e}_{2} \cdot \boldsymbol{\sigma}\right)\right] \Pi_{\nu},
\end{aligned}
$$

indicating the first order coupling among the various operators. The notation $J_{\mu}=$ $\frac{1}{2} \delta^{\alpha \beta} \Pi_{\alpha} \Pi_{\mu} \Pi_{\beta}$ has been introduced and all the functions are evaluated in $\vec{X}$. As expected from dimensional considerations $\mathcal{H}^{(1)}$ depends only on the first order derivatives of the filed. It is nevertheless worthwhile to stress that the gradient of the magnetic-field-norm, $\operatorname{grad} b=\boldsymbol{\nabla} b$, appears only in the first term of the right hand side of this expression, all the remaining terms depending only on the quantities $\mathcal{A}, \boldsymbol{l}_{\mathbf{1}}$ and $\boldsymbol{l}_{\mathbf{2}}$ completely characterizing the intrinsic/extrinsic geometry of the magnetic line bundle $\mathcal{M}$. To a large amount, therefore, the complication of this expression is produced by the variation of direction of the magnetic field, that is, by the nontrivial geometry of $\mathcal{M}$. It is not yet time to comment on the structure of $\mathcal{H}^{(1)}$. First of all, it is in fact necessary to operate a suitable unitary transformation separating the zero order fast motion from the other degrees of freedom, that is diagonalizing the the spin term $\boldsymbol{e}_{3} \cdot \boldsymbol{\sigma}$. This will produce a modification of the first order term of the adiabatic expansion. Secondly, it is possible to drastically simplify the form of $\mathcal{H}^{(1)}$ by operating a suitable first order unitary transformation. The strategy is nothing else than the quantum equivalent of the so called averaging transformation of classical mechanics and results of great help in shading light on the physical content of (31).

\section{Quantum averaging transformations}

A well known strategy in dealing with the adiabatic separation of fast and slow variables in classical mechanics consists in performing a series of successive canonical transformations (the averaging transformations) separating, order by order in some adiabatic parameter, the rapid oscillation of the system from its slow averaged motion. The analysis depending essentially on the canonical structure of the problem generalizes immediately to quantum 
mechanics, the canonical transformations being replaced by suitable unitary transformations. The full adiabatic expansion describing the motion of a spin degree of freedom adiabatically driven by external parameters has been obtained along these lines by M.V. Berry [16] while R.G. Littlejohn and S. Weigert [4] employed the method in discussing the first adiabatic corrections to the semiclassical motion of a neutral spinning particle in an inhomogeneous magnetic field. We shall consider therefore a set of unitary operators

$$
U^{(n)}=\exp \left\{i l_{B}^{n} \mathcal{L}^{(n)}\right\}
$$

$n=0,1, \ldots$ such that fast and slow/very-slow degrees of freedom separate up to $\mathcal{O}\left(l_{B}{ }^{n+1}\right)$

in the Hamiltonian obtained by the successive application of $U^{(0)}, U^{(1)}, \ldots, U^{(n)}$. Whereas in classical mechanics it is natural to consider the averaging transformation as defining new canonical variables, in quantum mechanics it appears more convenient to keep the canonical operators fixed and transform the Hamiltonian.

\section{Zero-order transformation}

The zero order separation of the fast and slow/very-slow motion requires the diagonalization of the spin term $g b(\vec{X})\left(\boldsymbol{e}_{3}(\vec{X}) \cdot \boldsymbol{\sigma}\right)$ of Hamiltonian (30). Denoting by $\rho_{i}{ }^{j}(\vec{x})$ the infinitesimal generator of the rotation $R_{i}{ }^{j}(\vec{x})$ bringing the fixed frame $\{\hat{\boldsymbol{x}}, \hat{\boldsymbol{y}}, \hat{\boldsymbol{z}}\}$ into the adapted frame $\left\{\boldsymbol{e}_{1}(\vec{x}), \boldsymbol{e}_{2}(\vec{x}), \boldsymbol{e}_{3}(\vec{x})\right\}, R_{i}{ }^{j}=\left(\mathrm{e}^{\rho}\right)_{i}{ }^{j} \equiv \delta_{i}^{j}+\rho_{i}{ }^{j}+\frac{1}{2} \rho_{i}{ }^{k} \rho_{k}{ }^{j}+\ldots$, the aim is achieved by choosing

$$
\mathcal{L}^{(0)}=-\frac{1}{2} \varepsilon^{i j k} \rho_{i j}(\vec{X}) \sigma_{k}
$$

the matrix $\rho_{i j}=\rho_{i}{ }^{j}$ being evaluated in the guiding center operators $\vec{X}$. Because the commutation relations (24) the operator $U^{(0)}$ commute with $\Pi_{1}, \Pi_{2}$ and therefore with $J$, produces $\mathcal{O}\left(l_{B}\right)$ terms when commuting with $\Pi_{3}$ and $\mathcal{O}\left(l_{B}{ }^{2}\right)$ terms when commuting with functions of $\vec{X}$. In evaluating the new Hamiltonian $\mathcal{H}^{\prime}=U^{(0)} \mathcal{H} U^{(0)}{ }^{\dagger}=\mathcal{H}^{(0)^{\prime}}+l_{B} \mathcal{H}^{(1)^{\prime}}+\ldots$ up to terms of order $l_{B}^{2}$ we have therefore to worry only about the action of $U^{(0)}$ on $\boldsymbol{\sigma}$ and $\Pi_{3}$. A very rapid computation yields the transformation rule

$$
U^{(0)}\left(\boldsymbol{e}_{i} \cdot \boldsymbol{\sigma}\right) U^{(0)^{\dagger}}=\sigma_{i}+\mathcal{O}\left(l_{B}{ }^{2}\right)
$$

while the action of $U^{(0)}$ on $\Pi_{3}, U^{(0)} \Pi_{3} U^{(0)^{\dagger}}=\Pi_{3}+U^{(0)}\left[\Pi_{3}, U^{(0)^{\dagger}}\right]$, may be easily evaluated by computing the commutator in the original set of operators $\pi_{i} \mathrm{~s}$ and $x^{i} \mathrm{~s}$ and transforming back to adiabatic variables

$$
U^{(0)} \Pi_{3} U^{(0)^{\dagger}}=\Pi_{3}+l_{B}\left(\boldsymbol{e}_{3} \cdot \boldsymbol{l}_{\mathbf{2}}\right) \sigma_{1}-l_{B}\left(\boldsymbol{e}_{3} \cdot \boldsymbol{l}_{\mathbf{1}}\right) \sigma_{2}+l_{B}\left(\boldsymbol{e}_{3} \cdot \mathcal{A}\right) \sigma_{3}+\mathcal{O}\left(l_{B}{ }^{2}\right) .
$$


Subjecting $\mathcal{H}^{(0)}$ and $\mathcal{H}^{(1)}$ to the zero order averaging transformation $U^{(0)}$ and by using (34) and (35) we obtain the new adiabatic expansion

$$
\begin{aligned}
& \mathcal{H}^{(0)^{\prime}} / \hbar \omega_{B}=\frac{1}{2} \Pi_{3}^{2}+b\left(J+g \sigma_{3}\right) \\
& \mathcal{H}^{(1)^{\prime}} / \hbar \omega_{B}=-b^{-1 / 2} \varepsilon^{\mu \nu}\left(\boldsymbol{e}_{\mu} \cdot \nabla b\right)\left(\frac{2}{3} J_{\nu}+g \sigma_{3} \Pi_{\nu}\right)-\frac{2}{3} b^{1 / 2}\left(\boldsymbol{e}_{\mu} \cdot \mathcal{A}\right) J_{\mu} \\
+ & {\left[\frac{1}{2}\left(\boldsymbol{e}_{1} \cdot \boldsymbol{l}_{\mathbf{2}}-\boldsymbol{e}_{2} \cdot \boldsymbol{l}_{\mathbf{1}}\right) J-\left(\boldsymbol{e}_{3} \cdot \mathcal{A}\right)\left(J-\sigma_{3}\right)\right] \Pi_{3}+\frac{1}{4}\left(\boldsymbol{e}_{1} \cdot \boldsymbol{l}_{\mathbf{2}}+\boldsymbol{e}_{2} \cdot \boldsymbol{l}_{\mathbf{1}}\right)\left(\Pi_{1}^{2}-\Pi_{2}{ }^{2}\right) \Pi_{3} } \\
- & \frac{1}{4}\left(\boldsymbol{e}_{1} \cdot \boldsymbol{l}_{\mathbf{1}}-\boldsymbol{e}_{2} \cdot \boldsymbol{l}_{\mathbf{2}}\right)\left\{\Pi_{1}, \Pi_{2}\right\} \Pi_{3}+b^{-1 / 2}\left[\left(\boldsymbol{e}_{3} \cdot \boldsymbol{l}_{\mathbf{2}}\right) \Pi_{1}-\left(\boldsymbol{e}_{3} \cdot \boldsymbol{l}_{\mathbf{1}}\right) \Pi_{2}\right] \Pi_{3}{ }^{2} \\
+ & {\left[\left(\boldsymbol{e}_{3} \cdot \boldsymbol{l}_{\mathbf{2}}\right) \sigma_{1}-\left(\boldsymbol{e}_{3} \cdot \boldsymbol{l}_{\mathbf{1}}\right) \sigma_{2}\right] \Pi_{3}-g b^{1 / 2} \varepsilon^{\mu \nu}\left[\left(\boldsymbol{e}_{\mu} \cdot \boldsymbol{l}_{\mathbf{1}}\right) \sigma_{1}+\left(\boldsymbol{e}_{\mu} \cdot \boldsymbol{l}_{\mathbf{2}}\right) \sigma_{2}\right] \Pi_{\nu}, }
\end{aligned}
$$

All the functions are evaluated in $\vec{X}$. The fast and slow/very-slow motion are separated in this way in the zero order term of the adiabatic expansion but not in the first order term.

\section{First-order transformation}

The application of the first order averaging transformation $U^{(1)}$ to $\mathcal{H}^{\prime}$ produces the new Hamiltonian $\mathcal{H}^{\prime \prime}=U^{(1)} \mathcal{H}^{\prime} U^{(1)^{\dagger}}=\mathcal{H}^{(0)^{\prime}}+l_{B}\left(\mathcal{H}^{(1)^{\prime}}+i\left[\mathcal{L}^{(1)}, \mathcal{H}^{(0)^{\prime}}\right]\right)+\ldots$. It is then possible to simplify the first order term of the adiabatic expansion by choosing $\mathcal{L}^{(1)}$ in such a way that its commutator with $\mathcal{H}^{(0)^{\prime}}$ cancels as much terms as possible of $\mathcal{H}^{(1)^{\prime}}$. The analysis of the commutation relation involved and a little thought indicates that it is possible to annihilate all but not the third term of (37) by choosing

$$
\begin{gathered}
\mathcal{L}^{(1)}=-b^{-3 / 2}\left(\boldsymbol{e}_{\mu} \cdot \nabla b\right)\left(\frac{2}{3} J_{\mu}+g \sigma_{3} \Pi_{\mu}\right)+\frac{2}{3} b^{-1 / 2} \varepsilon^{\mu \nu}\left(\boldsymbol{e}_{\mu} \cdot \mathcal{A}\right) J_{\nu} \\
-\frac{1}{8} b^{-1}\left(\boldsymbol{e}_{1} \cdot \boldsymbol{l}_{\mathbf{2}}+\boldsymbol{e}_{2} \cdot \boldsymbol{l}_{\mathbf{1}}\right)\left\{\Pi_{1}, \Pi_{2}\right\} \Pi_{3}-\frac{1}{8} b^{-1}\left(\boldsymbol{e}_{1} \cdot \boldsymbol{l}_{\mathbf{1}}-\boldsymbol{e}_{2} \cdot \boldsymbol{l}_{\mathbf{2}}\right)\left(\Pi_{1}{ }^{2}-\Pi_{2}{ }^{2}\right) \Pi_{3} \\
-b^{-3 / 2}\left[\left(\boldsymbol{e}_{3} \cdot \boldsymbol{l}_{\mathbf{2}}\right) \Pi_{2}+\left(\boldsymbol{e}_{3} \cdot \boldsymbol{l}_{\mathbf{1}}\right) \Pi_{1}\right] \Pi_{3}^{2}+g^{-1} b^{-1}\left[\left(\boldsymbol{e}_{3} \cdot \boldsymbol{l}_{\mathbf{2}}\right) \sigma_{2}+\left(\boldsymbol{e}_{3} \cdot \boldsymbol{l}_{\mathbf{1}}\right) \sigma_{1}\right] \Pi_{3} \\
+\frac{g}{g^{2}-4} b^{-3 / 2}\left[\left(\boldsymbol{e}_{\mu} \cdot \boldsymbol{l}_{\mathbf{1}}\right)\left(2 \sigma_{1} \delta^{\mu \nu}-g \sigma_{2} \varepsilon^{\mu \nu}\right)-\left(\boldsymbol{e}_{\mu} \cdot \boldsymbol{l}_{\mathbf{2}}\right)\left(2 \sigma_{2} \delta^{\mu \nu}+g \sigma_{1} \varepsilon^{\mu \nu}\right)\right] \Pi_{\nu}
\end{gathered}
$$

The commutators of the zero order Hamiltonian (36) with the various terms of $\mathcal{L}^{(1)}$ yields the terms of (37) times the imaginary factor $i$, in such a way that they cancel in the 
new adiabatic expansion. All the terms but not the third. It is in fact immediate to convince that no operator may be found in such a way that its commutator with (36) produces a term proportional to $J \Pi_{3}$ and $\sigma_{3} \Pi_{3}$. The third therm of (37) may therefore not be removed from the adiabatic expansion representing a real first order coupling among fast and slow/very-slow motion and not a complication produced by a wrong choice of variables. Its relevance in the context of the classical guiding center motion has been first recognized by R.G. Littlejohn [1]. It is therefore not a surprise to re-find it in the discussion of the quantum guiding center dynamics. The quantum averaging method produces so the adiabatic expansion

$$
\begin{aligned}
& \mathcal{H}^{(0)^{\prime \prime}}=\mathcal{H}^{(0)^{\prime}} \\
& \mathcal{H}^{(1)^{\prime \prime}} / \hbar \omega_{B}=\left[\frac{1}{2}\left(\boldsymbol{e}_{1} \cdot \boldsymbol{l}_{\mathbf{2}}-\boldsymbol{e}_{2} \cdot \boldsymbol{l}_{\mathbf{1}}\right) J-\left(\boldsymbol{e}_{3} \cdot \mathcal{A}\right)\left(J-\sigma_{3}\right)\right] \Pi_{3},
\end{aligned}
$$

We observe that whereas the zero order terms (36) depends only on the magnetic-filednorm $b$ (other than on the commutation relations (24)) the first order term (40) is completely characterized by the Frobenius invariant (12), and by the magnetic filed lines torsion (11).

\section{Quantum guiding center dynamics and magnetic-induced geometric-magnetism}

The construction of a suitable set of non-canonical operators embodying the classically expected features of the motion of a charged particle in an inhomogeneous magnetic field and the quantum averaging method allow us to rewrite the Pauli Hamiltonian (13) in such a way that the fast degree of freedom - corresponding to the classical rotation of the particle around its guiding center - and the spin degree of freedom separate, up to terms of order $l_{B}{ }^{2}$, from the guiding center dynamics. The transformation to the adiabatic operators $\Pi_{i} \mathrm{~s}, X^{i} \mathrm{~s},(20)$ and (22), and application of the zero and first order quantum averaging operators, (33) and (38), produces in fact the Hamiltonian

$$
\mathcal{H} / \hbar \omega_{B}=\frac{1}{2} \Pi_{3}^{2}+b\left(J+g \sigma_{3}\right)-l_{B}\left[\tau\left(J-\sigma_{3}\right)-\frac{1}{2} \mathcal{F} J\right] \Pi_{3}+\mathcal{O}\left(l_{B}^{2}\right) .
$$

Disregarding terms of order higher than $l_{B}$ the operators $J$, representing the magnetic moment of gyration of the particle, and $\sigma_{3}$ are constant of motion of the system. Frozen 
the particle in one of its $J$ and $\sigma_{3}$ eigenstates Hamiltonian (41) describes therefore the corresponding guiding centre dynamics. As long as $\mathcal{O}\left(l_{B}{ }^{2}\right)$ are ignored $X^{1}$ and $X^{2}$ appear as non-dynamical external adiabatic parameters and only the $\Pi_{3}-X^{3}$ degree of freedom, representing in the classical limit the drift of the particle along the magnetic field lines, is dynamically relevant. To this order, therefore, the quantum guiding center dynamics is described by a one degree of freedom Hamiltonian given by the sum of the kinetic energy $\Pi_{3}^{2} / 2$ and of an effective potential proportional to $b(\vec{X}) . \Pi_{3}$ being a slow variable, that is of the same magnitude of the first adiabatic correction, the order $l_{B} \operatorname{term}\left[\tau\left(J-\sigma_{3}\right)-\right.$ $\mathcal{F} J / 2] \Pi_{3}$ may be identified as a magnetic-like interaction and reabsorbed in the zero order Hamiltonian as a gauge potential. The guiding center Hamiltonian rewrites in this way in the familiar form

$$
\mathcal{H} / \hbar \omega_{B}=\frac{1}{2}\left(\Pi_{3}-l_{B} A(\vec{X})\right)^{2}+V(\vec{X})+\mathcal{O}\left(l_{B}{ }^{2}\right),
$$

with

$$
\begin{aligned}
A(\vec{X}) & =\left(J-\sigma_{3}\right) \tau(\vec{X})-\frac{J}{2} \mathcal{F}(\vec{X}), \\
V(\vec{X}) & =\left(J+g \sigma_{3}\right) b(\vec{X}) .
\end{aligned}
$$

As it might be expected from the general discussion of section 3 the magnetic filed line torsion $\tau=\boldsymbol{e}_{3} \cdot \mathcal{A}$ appears as (a part of) a gauge potential in the effective slow dynamics, taking into account the anholonomy produced by the non trivial parallel transport of the magnetic line bundle $\mathcal{M}$. Maybe unexpected, at least form this point of view, is the contribution given by the Frobenius invariant. Let us in fact compare the guiding center motion of a charged particle along a magnetic filed line with the propagation of light in a coiled optical fiber [19] or to the motion of an electron constrained on a twisted line [20]. In both cases - sharing the same mathematical background - the adiabatic dynamics is coupled with an effective vector potential proportional to the torsion of the optical fiber or of the twisted line. The analogue contribution appears in the guiding center motion, $(J-$ $\left.\sigma_{3}\right) \tau(\vec{X})$, but it is not the whole story. The particle being not homogeneously constrained in the neighborhood of a line, it results sensible to the variation of the geometry in the magnetic field lines surrounding the one on which the guiding center si located. If all the field lines would have the same geometry, the foliation of $R^{3}$ in terms of them would be trivial, the Frobenius invariant zero and the situation analogue the examples above. The geometry of this foliation being in general non trivial it yields a further contribution to the gauge potential $A(\vec{X})$ proportional to the Frobenius invariant, $J \mathcal{F}(\vec{X}) / 2$. It is 
obviously not possible, in the general case, to remove the gauge potential (43) by means of a suitable choice of gauge.

In order to make the identification of (42) with a one - two, if we want to consider $X^{1}$, $X^{2}$ as dynamical - degree of freedom Hamiltonian complete it is necessary to replace the $\Pi_{i} \mathrm{~s}, X^{i} \mathrm{~s}$ by a set of canonical operators. The task is achieved by introducing a Darboux coordinate frame $\mathrm{x}^{i}=\mathrm{x}^{i}(\vec{x}), i=1,2,3$, bringing the closed skew symmetric two-form $b_{i j}(\vec{x})$ in the canonical form,

$$
b_{k l}(\vec{x}) \frac{\partial x^{k}}{\partial \mathrm{x}^{i}} \frac{\partial x^{l}}{\partial \mathrm{x}^{j}}=\varepsilon_{i j}
$$

$\left(\mathrm{x}^{1}(\vec{x}), \mathrm{x}^{2}(\vec{x})\right.$ may be identified with a pair of Euler potentials [18] for the magnetic filed $\boldsymbol{b}(\vec{x}), \boldsymbol{\nabla} \mathrm{x}^{1} \wedge \boldsymbol{\nabla} \mathrm{x}^{2}=\boldsymbol{b}$, while $\mathrm{x}^{3}(\vec{x})$ with the arc length of the magnetic filed lines). Defining $\mathrm{X}^{i}=\mathrm{x}^{i}(\vec{X}), i=1,2,3$ we get the canonical commutation relations $\left[\Pi_{3}, \mathrm{X}^{i}\right]=-i l_{B} \delta_{3}^{i}$ and $\left[\mathrm{X}^{i}, \mathrm{X}^{j}\right]=-i l_{B}{ }^{2} \varepsilon^{i j}$ allowing the identification of the operators describing the slow and the very-slow degrees of freedom. It is in principle possible to start from the beginning by introducing such curvilinear coordinates in $R^{3}$ and work out the problem by using a canonical set of operators [17. Nevertheless, whereas the existence of a Darboux coordinate frame is always guaranteed by Darboux theorem, it is hardly ever possible to find it explicitly and to proceed to the construction of the $\mathrm{X}^{i} \mathrm{~s}$. For this reasonthought the $\Pi_{i} \mathrm{~s}, \mathrm{X}^{i} \mathrm{~s}$ appear as the most natural variables for the problem - the explicit construction of a set of non canonical operators appears as a better strategy.

\section{Conclusions}

The main difficulty in addressing the separation of fast and slow degrees of freedom in the study of an adiabatic system consist generally in finding out a suitable set of variables adapting with sufficient accuracy to the different time scale behaviours of the system. Starting from the homogeneous case and the canonical commutation relations (5) we showed how the analysis of the canonical structure of a charged spinning particle moving in an external inhomogeneous magnetic field leads naturally to the construction - as power series in the magnetic length $l_{B}$ - of a suitable set of non-canonical operators allowing to systematically take into account the coupling between spatial and spin degrees of freedom. The new variables fulfil the very compact commutation relations (24) clearly displaying

\footnotetext{
${ }^{4}$ The introduction of Darboux coordinate would produce automatically the framing of $T_{\vec{x}} R^{3}$ by means of an adapted frame $\left\{\boldsymbol{e}_{1}(\vec{x}), \boldsymbol{e}_{2}(\vec{x}), \boldsymbol{e}_{3}(\vec{x})\right\}$. Our method, on the other hand, consists in adapting the frame of $T_{\vec{x}} R^{3}$ without introducing the curvilinear coordinates.
} 
the dependence of the canonical structure on the norm and direction of the external magnetic field. In terms of the new operators the Pauli Hamiltonian rewrites as a power series in the adiabatic parameter $l_{B}$ which may be brought in a particular simple form by operating suitable unitary transformations. In this way the fast degree of freedom of the system representing classically the rapid rotation of the particle around the guiding center and the spin are separated from the remaining degrees of freedom up to terms of order $l_{B}^{2}$. The resulting effective guiding center dynamics displays geometric-magnetism: the coupling with the geometry induced gauge potential (43), depending on the magnetic filed lines torsion (11) and on the Frobenius invariant (12), and with the scalar potential (44), proportional to the magnetic field norm. This completely extend to the quantum domain the previous classical treatments of the problem showing that the anholonomy first studied by R.G. Littlejohn in the classical guiding center theory plays an equivalent role in the discussion of the quantum problem. It is a feature of the canonical structure of the system after all. The geometrical mechanism responsible for the appearance of induced gauge structures has also been analyzed in some detail and formalized in the geometry of the magnetic line bundle $\mathcal{M}$.

In concluding we observe that our discussion gives in some sense the solution of only half of the problem. The guiding center dynamics is still characterized by the presence of a fast and a slow time scale (slow $\rightarrow$ fast, very-slow $\rightarrow$ slow) and is therefore amenable to a treatment by means of adiabatic techniques. Nevertheless, the remaining problem is not of a so deep geometrical nature as the original one and is probably not capable of a treatment in general terms.

\section{Acknoledgments}

It is a genuine pleasure to thank M.V. Berry for hospitality and a very stimulating "few days discussion about fast and slow" in Bristol. I am also indebted with J.H. Hannay, E. Onofri and J.M. Robbins for very helpful discussions on related topics. 


\section{References}

[1] R.G. Littlejohn, J. Math. Phys. 12, 2445 (1979); Phys. Fluids. 29, 1730 (1981); J. Plasma Phys. 29, 111 (1983); in Contemporary Mathematics Vol.28, 151, J.E. Marsden ed. , (American Mathematical Society, 1984); Phys. Rev. A38, 6034 (1988)

[2] M.V. Berry, in Fundamental Aspects of Quantum Theory, 267, V. Gorini and A. Frigerio eds. (Plenum, New York, 1986)

[3] Y. Aharonov and A. Stern, Phys. Rev. Lett. 69, 3593 (1992)

[4] R.G. Littlejohn and S. Weigert, Phys. Rev. A48, 924 (1993)

[5] T.G. Northrop, The Adiabatic Motion of Charged Particles (Interscience, New York, 1963)

[6] P. Maraner, J. Phys. A: Math. Gen. 29, 2199 (1996)

[7] M.V. Berry, Proc. Roy. Soc. London A392, 45 (1984); in Geometric Phases in Physics, A. Shapere and F. Wilczek eds. , (World Scientific, Singapore, 1989)

[8] H. Kuratsuji and S. Iida, Prog. Theo. Phys. 74, 439 (1985)

[9] J. Moody, A. Shapere and F. Wilczek, Phys. Rev. Lett. 56, 893 (1986)

[10] R. Jackiw, Comm. At. Mol. Phys. 20, 71 (1988)

[11] A. Shapere and F. Wilczek, Geometric Phases in Physics (World Scientific, Singapore, 1989)

[12] M.V. Berry and J.M. Robbins, Proc. Roy. Soc. London A442, 641 (1993); Proc. Roy. Soc. London A442, 659 (1993)

[13] B. Felsager and J.M. Leinaas, Nuc. Phys. B166, 162 (1980)

[14] F. Gliozzi, Nuc. Phys. B141, 379 (1978)

[15] M. Spivak, A Comprehensive Introduction to Differential Geometry, Vol.4, (Publish or Perish, Houston, 1975)

[16] M.V. Berry, Proc. Roy. Soc. London A414, 31 (1987) 
[17] C.S. Gardner, Phys. Rev. 115, 791 (1959)

[18] D.P. Stern, Am. J. Phys. 38, 494 (1970)

[19] R.Y. Chiao and Y.S. Wu, Phys. Rev. Lett. 57, 933 (1986); A. Tomita and R.Y. Chiao, Phys. Rev. Lett. 57, 937 (1986)

[20] S. Takagi and T. Tanzawa, Prog. Theoret. Phys. 87, 561 (1992) 